Recepción: 13 4/ 05 / 2018

Aceptación: 27 / 07 / 2018

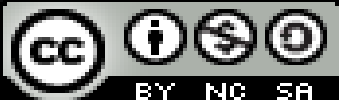

Ciencias de la educación

Publicación: 05 / 10 / 2018

Artículo de investigación

\title{
Aplicación de la técnica educativa ExamTime para aprendizaje de Botánica
}

\author{
Application of the educational technique ExamTime for learning Botany
}

Aplicação da técnica educacional Exame para aprender Botânica

Luís A. Mera-Cabezas I

lmera@unach.edu.ec

Correspondencia:1mera@unach.edu.ec

\footnotetext{
${ }^{I}$ Magister en Desarrollo de la Inteligencia y Educación, Licenciado en Ciencias de la Educación Profesor de Biología Química y Laboratorio, Docente de la Universidad Nacional de Chimborazo, Riobamba, Ecuador.
} 


\section{Resumen}

Actualmente la alfabetización tecnológica es una prioridad y la desvinculación entre la ciencia y la tecnología crea brechas en la educación que impiden al estudiante experimentar las ventajas de la aplicación de TIC's al momento de aprender. De ahí que el objetivo de esta investigación es demostrar si la aplicación de la técnica educativa ExamTime contribuye en el aprendizaje de Botánica, en los estudiantes de segundo y tercer semestre de la carrera de Biología, Química y Laboratorio de la UNACH, la metodología utilizada fue cuasi experimental, se realizó un trabajo comparativo entre grupo experimental y un grupo control para comprobar la hipótesis se usó la diferencia de Medias, donde se obtuvo un rendimiento académico del grupo experimental de 8,64 en relación al grupo control de 5,06. Se evidenció que al incluir las TIC's en el proceso educativo se consigue la construcción de aprendizajes significativos estimulando la producción intelectual, la creación de secuencias didácticas y generación de nuevos conocimientos.

Palabras clave: ExamTime; recursos tecnológicos; aprendizaje; TIC.

\section{Abstract}

The most important challenges at the educational level are framed in the fulfillment of national and international quality standards, where technological literacy is a priority and the separation between science and technology creates gaps in education that prevent the student from experiencing the advantages of the application of ICTs when learning. Hence, the objective of this research is to demonstrate whether the application of the educational technique ExamTime contributes to the learning of botany, students of the First Semester, Andean Agricultural Career, the Higher Technological Institute "Jatun Yachay Wasi", June - October 2016, given that through it, content is organized in a logical manner, it develops the capacity for analysis and synthesis and structures its self-evaluation dynamically. In the development of this research, the methodology used was quasi-experimental, based on a bibliographic analysis including the review of similar works at national and international level, being able to work with an experimental group and a control group facilitated the comparative study, the technique of data collection was the checklist allowing to quantify the data and check the hypothesis based on the calculation of the difference of means, where an academic performance of the experimental group of 8.64 was obtained in relation to the control group of 5.06. From the results obtained, it was evidenced that by 
including ICTs in the educational process, the construction of meaningful learning is achieved by stimulating intellectual production, the creation of didactic sequences and the generation of new knowledge. Additionally, an alternative proposal was developed the Student's Guide for Botany Learning through the application of the ExamTime Educational Technique.

Key words: ExamTime; technological resources; learning, ICT.

\section{Resumo}

Atualmente, a alfabetização tecnológica é uma prioridade e a separação entre ciência e tecnologia cria lacunas na educação que impedem o aluno de experimentar as vantagens de usar as TICs ao aprender. Daí o objetivo desta pesquisa é demonstrar se a aplicação de ExamTime tecnologia educacional ajuda em aprender Botânica, no semestre de segunda e terceira de estudos de Biologia, Química e Laboratório UNACH, a metodologia utilizada foi, o trabalho experimental comparativo entre quase grupo experimental e um grupo controlo foi realizado para testar a hipótese de diferença média, onde um rendimento escolar do grupo experimental em relação ao controlo 8,64 5,06 grupos foi utilizado foi obtido. Evidenciou-se que, ao incluir as TIC no processo educativo, a construção de aprendizagens significativas é conseguida estimulando a produção intelectual, a criação de sequências didáticas e a geração de novos conhecimentos.

Palavras chave: ExamTime; recursos tecnológicos; aprendizagem TIC.

\section{Introducción}

Actualmente, las tecnologías de la información y comunicación (TIC’s), se han convertido rápidamente en un factor imprescindible en el desarrollo de los países dando paso a la denominada "Sociedad de la información y conocimiento"; esto se debe principalmente a un invento que apareció en el año de 1969, el Internet.

Siendo misión de la Universidad Nacional de Chimborazo, formar profesionales emprendedores, con bases científicas y axiológicas, que contribuyan en la solución de los problemas del país. (Universidad Nacional de Chimborazo, 2016), es inevitable aportar soluciones a problemas específicos del entorno; generando conocimiento a través de la innovación, con personal idóneo, comprometido institucional y socialmente, de allí que la formación del postgrado constituye uno de los aspectos más trascendentales de la universidad. 
Ésta investigación, es el resultado de una novedad científica de actualidad que requirió ser analizada, pues ExamTime es un recurso educativo versátil que permite crear diferentes tipos de materiales educativos y compartirlos fácilmente en la red. Además, los profesionales de cuarto nivel tienen la responsabilidad social de ejecutar investigaciones que optimicen el proceso de enseñanza - aprendizaje y de esta manera logren el desarrollo de competencias que permitan al estudiante mejorar el aprendizaje de Botánica mediante el uso de recursos tecnológicos aplicados a la educación.

Existen muchos conceptos acerca de que es el proceso enseñanza-aprendizaje, y se puede decir que básicamente es el proceso por el cual se transmiten conocimientos que pueden ser específicos o generales sobre un determinado tema, y que incide directamente en el rendimiento académico del estudiante.

Los estilos de aprendizaje tienen que relación con las actitudes personales basadas en su experiencia académica y profesional. El uso de las TIC en educación ha sufrido un avance muy interesante desde hace casi ya 25 años que se creó la primera página de Internet un 23 de Agosto de 1991, y junto con ello se abrió una ventana virtual al mundo, y mediante la cual nos hemos podido conectar, educar, informar y aprender, por lo tanto los estilos de enseñanza-aprendizaje que se emplea en el aula de clase no pueden quedar marginados de dicho avance tecnológico, por lo tanto deben adaptarse a usar las TIC's en el proceso de enseñanza con características significativas tales como:

Ampliar el campo del conocimiento y las posibilidades de aprendizaje

Eliminar barreras espacio-temporales y permitir el acceso a la información.

Facilitar el trabajo colaborativo y el autoaprendizaje.

Desarrollar la interactividad y la flexibilidad en el proceso de aprendizaje.

Analizando lo expuesto, el uso de las TIC no puede quedar al margen del proceso educativo, pues si el objetivo de la educación es preparar individuos capaces de investigar y generar conocimiento, una de las maneras es incluir la tecnología en el proceso de aprendizaje, además, actualmente el Internet juega un papel importante en el desarrollo del individuo. 
Las tecnologías de la información y comunicación, más conocidas por sus siglas TIC's; constituyen uno de los recursos educativos tecnológicos actuales, cuya inserción en educación cada vez es mayor gracias a las múltiples bondades que estas ofrecen y los beneficios que aportan al proceso de enseñanza - aprendizaje.

Desde la década de los 90 hasta la actualidad, la tendencia al empleo de las TIC's, ha aumentado vertiginosamente, pues la sociedad de la información y conocimiento no concibe su existencia sin ellas, más aún cuando está íntimamente relacionado con el Internet, por tal motivo hoy por hoy se han creado un sinnúmero de artefactos electrónicos que mantienen al mundo "conectado", permitiendo su interacción con la sociedad, sin importar tiempo, espacio o distancia existente.

Para Aguaded y Cabero, las TIC's son: "Tecnologías que giran en torno a tres medios básicos: la informática, la microelectrónica y las telecomunicaciones; pero giran, no sólo de forma aislada, sino lo que es más significativo de manera interactiva e interconexionadas, lo que permite conseguir nuevas realidades comunicativas” (Aguaded Gómez \& Cabero Almenara, 2002)

Las TIC's, otorgan al proceso educativo una nueva oportunidad, donde los docentes deben abandonar actividades tradicionales de enseñanza, ampliar sus horizontes y emplear nuevas actividades y recursos de aprendizaje, entre los que destacan los recursos digitales que pueden provenir de software educativos, como también los puede elaborar el docente e incluso el mismo estudiante. Una de las ventajas principales es que muchos de los recursos tecnológicos disponibles, son de acceso libre y contemplan la gratuidad, por lo que el docente y estudiante pueden hacer uso de ellos sin mayores complicaciones, un ejemplo de éstos recursos lo constituye ExamTime.

Las TIC's, son un conjunto de herramientas relevantes dentro del proceso educativo, sobre todo si nos enfocamos en el estudio de Botánica como Ciencia Básica en la formación profesional Agropecuario Andino, permitiéndonos realizar nuevos enfoques teóricos, metodológicos y proponiendo la investigación de contenidos y la sistematización del aprendizaje como ejes principales del proceso. (Cortellezzi, 2014).

La Botánica, debe favorecer el desarrollo del pensamiento científico de los estudiantes, partiendo desde una actitud positiva que le permita enfrentar los problemas académicos no solo con un 
simple conocimiento memorístico, sino por medio de ideas, datos, hechos, resultados o teorías que se han acumulado a lo largo de su proceso educativo.

Como herramientas que ayudan al aprendizaje de Botánica, es necesario enfocarnos, en aquellos recursos que dispone ExamTime y que facilitan el proceso de aprendizaje a través de la síntesis de contenidos elaboración de mapas mentales, la aprehensión del conocimiento mediante las fichas de estudio y la autoevaluación donde el desarrollo de test juega un papel importante. (Guffante, 2015).

ExamTime, reúne diversas herramientas de estudio online gratuitas, disponible para docentes y estudiantes, ya que a través de las actividades propuestas en la plataforma se puede crear, descubrir y compartir recursos de estudio como mapas mentales, fichas de estudio, apuntes interactivos y test online, impulsando el aprendizaje colaborativo, por medio de comunidades educativas virtuales, que permiten mejorar el proceso educativo, convirtiendo el aprendizaje en un proceso activo, dinámico, personalizado y adecuado a la tecnología actual.

\section{Metodología}

La población objeto de estudio son 46 estudiantes matriculados en el segundo y tercer semestre de la Carrera de Biología Química y Laboratorio. Se dividió a los estudiantes en dos grupos, uno de control y el otro experimental con el cual se utilizó ExamTime con la finalidad de aprovechar de manera significativa las múltiples ventajas que esta herramienta proporciona, se desarrolló por parte de los estudiantes un conjunto de actividades de contenido muy variado en relación al microcurriculo vigente de la asignatura de botánica junto con una recopilación de ideas prácticas, descritas claramente y con ilustraciones adecuadas.

El trabajo estuvo orientado hacia los estudiantes que desean vincular las TIC's en su proceso de aprendizaje de Botánica, y que permite desarrollar de manera responsable el trabajo autónomo y, de este modo, alcanzar los objetivos de aprendizajes de la asignatura mencionada; la herramientas educativas fue aplicada por unidades temáticas de acuerdo al Plan Analítico de la Asignatura de Botánica, donde además de construir conocimientos se fortaleció el trabajo autónomo a través del desarrollo de la creatividad, la capacidad de análisis y síntesis y la autoevaluación constante. Los temas de cada una de las unidades temáticas desarrolladas usando el ExamTime como recurso 
didáctico que potenció un trabajo autónomo en los alumnos se encuentran distribuidas de la siguiente manera:

Una vez desarrolladas las actividades mencionadas se procedió a evaluar los resultados de aprendizaje de los estudiantes, comparando el rendimiento académico obtenido por el grupo de control y el experimental.

\section{Resultados y Discusión}

La estadística aplicada en la comprobación de la hipótesis de investigación fue el análisis de promedios a través de la comparación de medias: Hi: X1 > X2 Ho: X1= X2.

\section{Tabla 1}

Promedio de rendimiento en la asignatura de Botánica

\begin{tabular}{ll}
\hline Grupo experimental & Grupo de control \\
\hline 8,56 & $\mathbf{5 , 1 0}$ \\
\hline
\end{tabular}

Fuente: cuestionarios aplicados

Como 8,56 > 5,10 se rechaza la Hipótesis Nula y se procede a la aceptación de la Hipótesis de investigación: La aplicación de la técnica educativa ExamTime mediante la utilización de mapas mentales contribuye en el aprendizaje de Botánica de los estudiantes segundo y tercer semestre de la carrera de Biología Química y Laboratorio.

Se procedió a tabular los resultados de las evaluaciones aplicadas a los estudiantes en base a criterios que permitieron evidenciar los logros de aprendizajes declarados en el silabo.

¿El estudiante explica las divisiones de la Botánica de manera clara, jerarquizando contenidos e incluyendo imágenes adecuadas?

\section{Tabla 2}

Respuesta a la pregunta 1 de la encuesta aplicada a los estudiantes de segundo y tercer semestre

\begin{tabular}{|l|c|}
\hline \multicolumn{1}{|c|}{ PARALELO } & PROMEDIO \\
\hline Grupo experimental & 7,79 \\
\hline Grupo Control & 5,78 \\
\hline
\end{tabular}

Fuente: cuestionarios aplicados 
Se evidenció que el grupo experimental consiguió mejor desempeño al explicar las divisiones de la Botánica de manera clara, por otra parte, el desempeño del grupo de control al aplicar la Técnica Educativa ExamTime en la elaboración de mapas mentales, no fue satisfactorio, ya que el desconocimiento de la técnica impidió la explicación de las divisiones de la Botánica de manera clara, dificultando su fácil seguimiento y comprensión, además, no se jerarquizó los contenidos e incluyó imágenes, esto repercutió de manera significativa en la construcción del conocimiento, motivo por el cual no se alcanzó el dominio del aprendizaje requerido.

¿El estudiante explica la clasificación de las plantas sagradas de manera clara, jerarquizando contenidos e incluyendo imágenes adecuadas?

\section{Tabla 3}

Respuesta a la pregunta 2 de la encuesta aplicada a los estudiantes de segundo y tercer semestre

\begin{tabular}{|l|c|}
\hline \multicolumn{1}{|c}{ PARALELO } & PROMEDIO \\
\hline Grupo experimental & 8,51 \\
\hline Grupo Control & 538 \\
\hline
\end{tabular}

Fuente: cuestionarios aplicados

Se observó que los estudiantes del grupo experimental, presentaron mayor facilidad para organizar la información de manera adecuada, explicando la clasificación de las plantas sagradas de manera clara, jerarquizando contenidos e incluyendo imágenes adecuadas, en cuanto al desempeño del grupo de control, éste presentó dificultad al momento de organizar la información de manera adecuada, no ubicaron la palabra central ni la asocian correctamente con el tema, no dieron relevancia a las ideas principales y secundarias, ni representaron conceptos importantes en relación a las plantas sagradas y sus divisiones, obteniendo un promedio de 5,38, es así que éste grupo no alcanzó a dominar el aprendizaje requerido en función de organización de la información y la aplicación adecuada de este recurso.

¿El estudiante explica la clasificación de las plantas medicinales de manera clara, jerarquizando contenidos e incluyendo imágenes adecuadas? 


\section{Tabla 4}

Respuesta a la pregunta 3 de la encuesta aplicada a los estudiantes de segundo y tercer semestre

\begin{tabular}{l|c|}
\hline \multicolumn{1}{c}{ PARALELO } & PROMEDIO \\
\hline Grupo experimental & 8,46 \\
\hline Grupo Control & 4,78 \\
\hline
\end{tabular}

Fuente: cuestionarios aplicados

En base a los datos obtenidos, se pudo observar que los estudiantes del grupo experimental, incluyeron imágenes pertinentes en el mapa mental y las relacionaron de manera adecuada al conocimiento adquirido de las plantas medicinales y sus divisiones, aplicando de forma satisfactoria este recuso obteniendo un promedio general de 8,46, alcanzando el aprendizaje requerido en la ejecución del mismo. Al analizar los datos del grupo de control, éste presentó inconvenientes para incluir imágenes pertinentes en el mapa mental y relacionarlas de manera adecuada al conocimiento adquirido de las plantas medicinales y sus divisiones, no aplicaron de manera satisfactoria este recuso, consiguiendo un promedio de 4,78 por lo que no se logró dominar el aprendizaje requerido en la ejecución del trabajo encomendado.

¿El estudiante explica la clasificación de las plantas industriales de manera clara, jerarquizando contenidos e incluyendo imágenes adecuadas?

\section{Tabla 5}

Respuesta a la pregunta 4 de la encuesta aplicada a los estudiantes de segundo y tercer semestre

\begin{tabular}{l|c|}
\hline \multicolumn{1}{|c}{ PARALELO } & PROMEDIO \\
\hline Grupo experimental & 9,38 \\
\hline Grupo Control & 4,97 \\
\hline
\end{tabular}

Fuente: cuestionarios aplicados

Se evidenció que el grupo experimental, obtuvo un desempeño óptimo al aplicar la Técnica Educativa ExamTime en la elaboración de mapas mentales con relación a la clasificación de las plantas industriales de manera clara, jerarquizando contenidos e incluyendo imágenes adecuadas ya que utilizó conectores adecuados para relacionar la información de manera secuencial y lógica, además, logró la construcción y conexión adecuada de la información y alcanzó el aprendizaje requerido con un promedio de 8,46. Por otra parte, el desempeño del grupo de control al aplicar la Técnica Educativa ExamTime en la elaboración de mapas mentales con relación a la 
clasificación de las plantas industriales de manera clara, jerarquizando contenidos e incluyendo imágenes adecuadas, no fue satisfactoria, pues no utilizó conectores adecuados para relacionar la información de manera secuencial y lógica, motivo por el cual no alcanzó el dominio del aprendizaje requerido ya que obtuvo un promedio de 4,78 .

¿El estudiante explica la clasificación de las plantas ornamentales de manera clara, jerarquizando contenidos e incluyendo imágenes adecuadas?

\section{Tabla 6}

Respuesta a la pregunta 5 de la encuesta aplicada a los estudiantes de segundo y tercer semestre

\begin{tabular}{|l|c|}
\hline \multicolumn{1}{|c}{ PARALELO } & PROMEDIO \\
\hline Grupo experimental & 8,64 \\
\hline Grupo Control & 4,61 \\
\hline
\end{tabular}

Fuente: cuestionarios aplicados

Del análisis de los datos obtenidos con el grupo experimental, se pudo evidenciar un desempeño óptimo al elaborar un mapa mental innovador, creativo, atractivo, jerárquico, acerca de la clasificación de las plantas ornamentales incluyendo imágenes adecuadas, además, logró la construcción del conocimiento obteniendo un promedio de 8,64, alcanzando el aprendizaje requerido a través de la aplicación de la Técnica Educativa ExamTime. Al mismo tiempo, el desempeño del grupo de control al aplicar la Técnica Educativa ExamTime en la elaboración de un mapa mental innovador, creativo, atractivo, jerárquico, acerca de la clasificación de las plantas ornamentales incluyendo imágenes adecuadas, no fue satisfactorio, pues no se logró la construcción y conexión adecuada de la información, obteniendo un promedio de 4,61, motivo por el cual no alcanzó el dominio del aprendizaje requerido.

¿Estructura la pregunta de manera clara, organizada, incluye elementos visuales adecuados y direcciona a la búsqueda de una respuesta específica, referente al tema de las algas?

\section{Tabla 7}

Respuesta a la pregunta 6 de la encuesta aplicada a los estudiantes de segundo y tercer semestre

\begin{tabular}{|l|c|}
\hline \multicolumn{1}{|c|}{ PARALELO } & PROMEDIO \\
\hline Grupo experimental & 8,23 \\
\hline Grupo Control & 5,27 \\
\hline
\end{tabular}

Fuente: cuestionarios aplicados 
Del análisis de los datos obtenidos con el grupo experimental, se pudo evidenciar que tienen facilidad para estructurar preguntas de manera clara, organizada acorde a la información de las algas, consiguiendo un desempeño muy bueno, logrando la reconstrucción del conocimiento, obteniendo un promedio de 8,23 y alcanzando el aprendizaje requerido a través de la aplicación de la técnica educativa ExamTime. Al mismo tiempo, el desempeño del grupo de control no fue adecuado, los estudiantes no estructuraron preguntas de manera clara, organizada acorde a la información de las algas, esto se debe principalmente al desconocimiento en cuanto a la utilización de la Técnica Educativa ExamTime, factor determinante a momento de la reconstrucción del conocimiento, obteniendo un promedio de 5,27, donde los aprendizajes requeridos no fueron alcanzados.

\section{Conclusiones}

La aplicación de la técnica educativa ExamTime mediante la utilización de mapas mentales contribuye en el aprendizaje de Botánica de los estudiantes del segundo y tercer Semestre, de la Carrera Biología Química y Laboratorio, al organizar los contenidos de manera lógica.

De acuerdo a los resultados obtenidos y expresados se evidencia que la aplicación de la técnica educativa ExamTime por medio de la creación de fichas de memoria favorece el aprendizaje de Botánica de los estudiantes ya que favorece el desarrollo de la capacidad de análisis y síntesis.

Se comprueba que la implementación de la técnica educativa ExamTime a través de la elaboración de test online mejora el aprendizaje de Botánica al estructurar autoevaluaciones dinámicas.

\section{Referencias Bibliográficas}

Aguaded Gómez, J. I., \& Cabero Almenara, J. (2002). Educar en red: Internet como recurso para la educación. México: Aljibe.

Cortellezzi, R. (12 de 04 de 2014). Administración nacional de educación pública. Recuperado de: http://www.anep. edu.uy

Guffante, R. (2015). Aplicación de la WEB 2.0 como estrategia metodológica para el aprendizaje de informática TICs dirigida a estudiantes del primer semestre de Psicología Educativa y Ciencias Sociales de la UNACH. Riobamba: Universidad Nacional de Chimborazo. 
Martínez, E., \& Sánchez, S. (17 de 07 de 2014). Proceso enseñanza aprendizaje. Recuperado de: http://www.uhu.es

Sáenz López, J. (2010). Utilización de las TIC en el proceso de enseñanza aprendizaje, valorando la incidencia real de las tecnologías en la práctica docente. Docencia e Investigación, pp. 183 204

Villa, C. (2013). Integración de las TIC (Tecnologías de la Información y Comunicación) en el currículo de los docentes de lengua y literatura realizado en las instituciones: escuela de educación básica 12 de febrero, colegio de bachillerato Manuela Garaicoa de Calderón, U. Loja: Universidad Técnica Particular de Loja. 